\title{
2016 American Association of Clinical Endocrinologists/ American College of Endocrinology Clinical Practice Guidelines on Postmenopausal Osteoporosis
}

Pauline M Camacho

Loyola University Osteoporosis and Metabolic Bone Disease Center, Maywood, Illinois, US

$\mathrm{T}$ he 2016 American Association of Clinical Endocrinologists/American College of Endocrinology Guidelines for postmenopausal osteoporosis provides comprehensive guidance on the diagnosis, evaluation and treatment of postmenopausal osteoporosis. A new diagnostic criterion which expands the diagnosis to patients with osteopenia and a high Fracture Risk Assessment Tool score has been added to the old diagnostic criteria. Recommendations on duration of bisphosphonate therapy are clearly outlined and are based on fracture risk stratification. The accompanying algorithm, which goes through all the steps from diagnosis to treatment and follow up is a handy guide for clinicians.

\section{Keywords}

Postmenopausal osteoporosis, American Association of Clinical Endorinologists/American College of Endocrinology Guidelines, bone mineral density, osteopenia, fracture risk

Disclosure: Pauline M Camacho has nothing to disclose in relation to this article. This article is a short opinion piece and has not been submitted to external peer reviewers. No funding was received for the publication of this article.

Authorship: All named authors meet the International Committee of Medical Journal Editors (ICMJE) criteria for authorship of this manuscript, take responsibility for the integrity of the work as a whole, and have given final approval to the version to be published.

open Access: This article is published under the Creative Commons Attribution Noncommercial License, which permits any noncommercial use, distribution, adaptation, and reproduction provided the original author(s) and source are given appropriate credit.

Received: October 25, 2016

Published Online: December 20, 2016

Citation: US Endocrinology, 2016;12(2):74-5

Corresponding Author: Pauline M Camacho, 2160 S. First Ave, Fahey Center Suite 137, Maywood, IL 60153, US. E: pcamach@|umc.edu
In September 2016, the latest American Association of Clinical Endocrinologists (AACE)/American College of Endocrinology (ACE) Guidelines for postmenopausal osteoporosis were published. This contains very relevant information on the diagnosis, and long term management of osteoporosis in postmenopausal women. Included in this version is a user-friendly algorithm which clearly outlines the approach to the choice of initial therapy, length of therapy, and what to do when treatment is not working.

The diagnostic criteria for osteoporosis is similar to that in the previous (2010) version of the guidelines. It is diagnosed based on the presence of a fragility fracture in the absence of another metabolic bone disorder; and a lumbar, total, femoral neck hip bone mineral density (BMD) T-score of -2.5 or lower (with the distal radius $33 \%$ as an alternate site). However, patients with osteopenia (T-score of -1.0 to -2.4 ) and a high fracture risk score using the Fracture Risk Assessment Tool (FRAX ${ }^{\circledR}$ ), may also be diagnosed with osteoporosis. This expanded criteria is consistent with the National Bone Health Alliance position statement on the clinical diagnosis of osteoporosis.

The choice of initial therapy would depend on the patient's fracture risk. Individuals with a higher fracture risk include those who are older, those who have prior fractures, those with very low T-scores, and those who are frail or are on glucocorticoid therapy. For higher fracture risk individuals, an injectable agent such as denosumab, teriparatide or zoledronic acid could be considered as initial therapy. Individuals at moderate risk may be started on alendronate, risedronate, denosumab or zoledronic acid. All four of these agents have proven fracture risk reduction in vertebral, non-vertebral and hip sites. Alternative drugs include ibandronate and raloxifene.

The recommended duration of therapy would also be based on the patient's fracture risk. Five years of oral bisphosphonates (BP) or three 
years of zoledronic acid is appropriate for moderate risk patients. For higher fracture risk patients, the recommended treatment duration is up to 10 years of oral BP or six years of zoledronic acid. Teriparatide therapy is limited to two years and drug holidays are not recommended for denosumab.

Bisphosphonate holidays end when there is a fracture, BMD declines beyond the least significant change (LSC) of the machine, or clinical fracture risk increases significantly. Bone turnover markers rising to pretreatment levels may also herald the end of the drug holiday.

The latest AACE/ACE Postmenopausal Osteoporosis Guidelines has updated recommendations on the diagnosis, initial and long-term management of postmenopausal osteoporosis patients. The osteoporosis algorithm, which will soon be available in app form is an essential guide for practicing clinicians. $\square$

1. Camacho PM, Petak SM, Binkley N, et al., American Association of Clinical Endocrinologists and American College of Endocrinology Clinical Practice Guidelines for the Diagnosis and Treatment of Postmenopausal Osteoporosis - 2016. Available at: www.aace.com/files/final-appendix-sept-7.pdf (accessed November 16, 2016). 